\title{
Erratum to: Dilepton production in proton-induced reactions at SIS energies with the GiBUU transport model
}

The Publishers

EDP Sciences, Società Italiana di Fisica and Springer-Verlag

Original article: Eur. Phys. J. A (2012) 48: 111, DOI: 10.1140/epja/i2012-12111-9

Received: 18 October 2012

Published online: 31 October 2012 - (C) Società Italiana di Fisica / Springer-Verlag 2012

\begin{abstract}
We present dilepton spectra from $p+p, d+p$ and $p+\mathrm{Nb}$ reactions at SIS energies, which were simulated with the GiBUU transport model in a resonance model approach. These spectra are compared to the data published by the HADES and DLS Collaborations. It is shown that the $\rho$ spectral function includes non-trivial effects already in elementary reactions, due to production via baryon resonances, which can yield large contributions to the dilepton spectrum. Dilepton spectra from nuclear reactions in the energy range of the HADES experiment are thus found to be sensitive also to properties of nucleon resonances in the nuclear medium.
\end{abstract}

For a technical incovenience, this paper has been published with only the first line of the abstract, which we publish herewith again.

The Publishers regret this mistake and deeply apologize to the authors. 